\title{
Psychometric properties of the Thai version of the Diabetes Distress Scale in diabetic seniors
}

\author{
This article was published in the following Dove Press journal: \\ Clinical Interventions in Aging \\ 16 August 2014 \\ Number of times this article has been viewed
}

\section{Kattika Thanakwang \\ Wantana Thinganjana \\ Roumporn Konggumnerd}

Institute of Nursing, Suranaree University of Technology, Nakhon Ratchasima, Thailand
Correspondence: Kattika Thanakwang Institute of Nursing, Suranaree University of Technology, III University Avenue, Tambon Suranaree Amphur Muang, Nakhon Ratchasima, Thailand, 30000 Tel +66 44223520

Fax +66 44223506

Email kattika@sut.ac.th
Background: The Diabetes Distress Scale (DDS) is an important measure of diabetes-related emotional distress that has been widely used in the Western world. In Thailand, there is a lack of reliable and valid scales for assessing distress levels in diabetes patients, specifically in older adults.

Objectives: The main objectives of this study were to adapt the DDS for use in Thai diabetic elderly and to evaluate its psychometric properties.

Methods: The 17-item DDS was linguistically adapted using forward-backward translation and administered to 170 diabetic patients $\geq 60$ years selected from diabetes outpatient clinics of four hospitals in Buriram, Thailand. Statistical analyses included exploratory factor analysis, internal consistency, convergent validity, and test-retest reliability.

Results: During factor analysis, a three-factor solution was found to be reasonable for the subdimensions of emotional and regimen-related burden (ten items), physician- and nurse-related distress (four items), and diabetes-related interpersonal distress (three items). The Cronbach's alpha coefficient for the total score was 0.95 and varied between 0.85 and 0.96 in the three subscales. The results provided evidence that supports the convergent validity of the Thai version of the DDS as well as its stability.

Conclusion: The Thai version of the DDS has acceptable psychometric properties. It enables assessment of diabetes-specific distress in elderly patients and has the advantage of being easy to use in both clinical and research settings.

Keywords: diabetes distress scale, psychometric properties, diabetes-related stress, diabetic elderly

\section{Introduction}

Diabetes is a chronic illness requiring substantial long-term treatment throughout a patient's lifespan. Individuals living with diabetes often suffer from lifelong selfmanagement regimens, involving significant changes in lifestyle such as diet, weight control, exercise, and other health-promoting behaviors, as well as adherence to complex medication regimens. ${ }^{1,2}$ It is not surprising that psychological distress is prevalent in diabetes patients, specifically in patients who are dependent on others and lack the resources and abilities for self-care.

Diabetes-related distress (DRD) is a syndrome comprised of multidimensional components such as worry, conflict, frustration, and discouragement that can accompany living with diabetes. ${ }^{3,4}$ Negative physical and psychological effects can be directly attributable to long-term suffering from diabetes-related emotional distress. There is some evidence that the negative effects of depression on diabetes, ${ }^{4,5}$ inability to self-manage, ${ }^{2}$ poor glycemic control, ${ }^{4,6,7}$ and ineffective treatment outcomes ${ }^{8}$ can be predicted by diabetic distress. 
Studies have shown that emotional distress, stress, and depression are significantly related to each other in chronically ill patients, particularly in diabetic patients. ${ }^{2,6}$ A study by Polonsky et al showed that DRD was significantly associated with depressive symptoms. ${ }^{4}$ DRD was found to be more prevalent than depression in individuals with diabetes, and more strongly associated with poor diabetes self-management and glycemic control compared to depression. ${ }^{3,9}$ However, DRD is often not recognized by health care professionals and thus ineffectively addressed by treatment strategies. ${ }^{10}$ As a result, emotional distress in diabetic individuals is an important issue that should concern health care professionals. ${ }^{6}$

Several instruments for measuring DRD have been developed such as the ATT39, ${ }^{11}$ the Questionnaire on Stress in Patients with Diabetes-Revised (QSD-R), ${ }^{12}$ and the Problem Areas in Diabetes Scale (PAID). ${ }^{2}$ However, these instruments have limitations. The ATT39 and QSD-R are relatively long ( 39 and 45 items, respectively) while the PAID is rather brief (20 items). Additionally, subscales have not been developed for these scales and some items are difficult to understand. ${ }^{4}$ To address these shortcomings, Polonsky et a ${ }^{4}$ developed the Diabetes Distress Scale (DDS) based on the QSD- ${ }^{12}$ and PAID. ${ }^{2}$ The DDS is a brief self-reporting measure of diabetes-related emotional distress that identifies a diabetic patient's concerns about disease management, emotional burden, social support, and health care accessibility. The DDS measures four distinct dimensions of distress: 1) regimen-related distress; 2) physician-related distress; 3) emotional burden; and 4) interpersonal distress. ${ }^{4,10}$

Distress over a diabetes regimen involves concerns and discouragement that patients perceive and/or encounter while self-managing their disease. For example, perceived difficulties in following or maintaining their diabetes management routine. Diabetes management requires vigilant and sustained adherence to a complex and coordinated treatment regimen comprised of health behavior changes such as exercise and use of prescribed medications. ${ }^{13}$ Physician-related distress includes concerns about access to health care and quality of care received, including concerns that recommendations provided by health care professionals could be incomplete. Emotional burden involves the negative mental and emotional aspects of living with diabetes. This includes feeling a variety of negative emotions such as despair, conflict, and fear-induced anger that result from thinking about the prospect of a lifetime of living with diabetes and feeling overwhelmed by the many demands. Finally, interpersonal distress, the result of day-to-day interactions or lack thereof with significant others involved in the patient's life, can contribute to DRD by limiting emotional support and making it more difficult to maintain a healthy lifestyle.

The DDS was developed to assess diabetic individuals and is used primarily in the USA., ${ }^{2,37}$ It has recently been translated and validated for use in several other countries including the People's Republic of China, ${ }^{14}$ Norway, ${ }^{15}$ and Denmark. ${ }^{16}$ However, these cross-cultural validated DDSs were conducted in general diabetes populations. There is no DRD instrument that focuses on older patients who are more likely to suffer from emotional distress resulting from living with diabetes as is found in other age groups. ${ }^{1,17,18}$ In Thailand, there is a lack of reliable and valid scales for accurate assessment of distress levels in diabetic patients. To date, no study has specifically focused on diabetes distress in older Thai adults. As a result, the main purpose of this study is to translate the original DDS for use in Thai-speaking diabetic seniors and evaluate its psychometric properties.

\section{Methods}

A two-stage design was used. The first stage consisted in the development of a Thai language version of the DDS (ThaiDDS) by adapting the original English version ${ }^{4}$ and then implementing a forward-backward translation process to test the validity of the content. The second stage involved testing the psychometric properties of the Thai-DDS by examining construct and convergent validity as well as internal consistency and test-retest reliability.

\section{Stage I: Translation procedure}

A procedure for translating the original English version of the DDS developed by Polonsky et $\mathrm{al}^{4}$ into a Thai language version was developed and implemented to ensure compliance with the original English version. The procedure consisted of the following: 1) contacting Dr Polonsky for his approval to use, and translate the DDS into a Thai language version; 2) ensuring that the forward translation process included two professional bilingual translators; and 3) holding a consensus meeting during which the translators and research team reviewed and agreed on a Thai version that best reflected the linguistic and conceptual matter of the original English version. ${ }^{4}$

Backward translation involved hiring a native Englishspeaking translator to translate the Thai version into an English version. ${ }^{5}$ Another consensus meeting was held, during which one native English speaker and two bilingual Thai researchers compared the translation with the original English version to check for conceptual discrepancies and semantic equivalence. These discussions helped streamline the translation cross-culturally and contributed to standardization of 
the Thai-DDS. ${ }^{6}$ The final step consisted of a review of the Thai version by an expert panel. The instrument was sent to five experts specializing in multidisciplinary areas relevant to the study who were asked to evaluate the validity of its content. These included one expert from each of the following areas of: gerontological nursing, geriatric medicine, diabetes, linguistics and culture, and instrument development. Examination of content validity was based on expert concurrence using the content validity index (CVI). ${ }^{19} \mathrm{CVI}$ identified the proportion of total items judged as valid content; ${ }^{20}$ the instrument was deemed valid when the CVI value was $\geq 0.80 .{ }^{19}$ The expert reviewers were also invited to suggest revised wordings of any items that seemed ambiguous, unclear, or inappropriate.

The average CVI score for the Thai-DDS was 0.92, indicating an acceptable content validity for the scale. Semantic, idiomatic, conceptual, and cultural equivalences were also evaluated by an expert panel of judges. Most of the 17-items required no modification. However, in the physician-related distress component, the experts suggested having a nurse accompany the doctor, since nurses, specifically nurse practitioners and diabetes specialist nurses, play a prominent role in the diabetic health services of Thailand's health care system. As a result, the term "doctor" was replaced with the term "doctor or nurse" in items $2,4,9$, and 15, confident that this change would facilitate understanding by study participants. After modification to the terminology, the Thai-DDS was initially pre-tested in 30 elderly diabetic patients who were receiving care at a hospital diabetes outpatient clinic. All participants stated they had no difficulties understanding the items, and most were able to complete the questionnaire in 15 minutes.

\section{Stage 2: Psychometric testing}

A significant field test using the final version of the ThaiDDS was conducted using a large number of elderly diabetic patients, and further psychometric testing was performed to determine the validity and reliability of the newly developed measure for DRD.

The sample included 170 diabetic patients $\geq 60$ years, with an additional requirement that each of the 17-items received at least ten responses. ${ }^{21}$ Thus, 170 responses were deemed acceptable for determining factor analysis and internal consistence reliability, as well as for testing convergent validity. Following the requirements for obtaining a convenience sample, we selected four community hospitals in Buriram Province, Thailand. Elderly diabetic patients visiting the diabetes outpatient clinics of these hospitals were invited to participate in the study. Inclusion criteria were: 1 ) $\geq 60$ years; 2) a diagnosis of diabetes with disease onset duration of $\geq 1$ years; 3 ) not currently suffering from severe disabilities, dementia, or psychiatric disorders; and 4) able to understand and speak Thai. For test-retest reliability, 30 diabetic seniors in one hospital in Nangrong District, Buriram Province were selected as participants based on various demographic characteristics (such as, sex, age, marital status, education, and income) to make the sample heterogeneous.

\section{Measures DDS}

The $\mathrm{DDS}^{4}$ has 17 -items and four dimensions, which include emotional burden (five items), regimen-related distress (five items), physician-related distress (four items), and diabetesrelated interpersonal distress (three items). The items are rated on a 6-point Likert scale ranging from 1 (no problems) to 6 (serious problems) based on the degree that each item is currently problematic for the participant. The original version of the DDS has been shown to be reliable (Cronbach's alpha $=0.93) .{ }^{4}$ The mean score for overall diabetes distress was calculated by dividing the total score by 17 , and each domain-specific subscale was calculated by dividing the total scores of the subscale by the number of questions associated with it. With a possible range of 1 to 6 , the overall DDS and each sub-component scale were evaluated using the following: a mean score of $<2$, indicating no distress; a mean score between 2-2.9, indicating moderate distress; and a mean score $\geq 3$, indicating high distress. ${ }^{22}$

\section{Thai-Geriatric Depression Scale}

The Thai Geriatric Depression Scale (GDS) was developed by the Train the Brain Forum Committee consisting of 29 individuals (ie, neurologists, psychiatrists, geriatricians, psychiatric nurses, and psychologists) from 14 institutions in Thailand such as the faculty of medicine of six universities, seven tertiary hospitals, and the Biomedical Instrumentation for Research and Development Centre. ${ }^{23}$ The Thai-GDS is a quick, easy, and accurate self-assessment screening test highly relevant to Thai culture. It is comprised of 30 items, with a possible score ranging from 0 to 30. The criteria for depression in Thai seniors according to Thai-GDS scores are as follow: a) normal, 0-12 points; b) mild depression, 13-18 points; c) moderate depression, 19-24 points; and d) severe depression, 25-30 points. The Thai-GDS has shown good reliability, with a Kuder-Richardson coefficient (K-R 20) of 0.93. The Thai-GDS was used to assess the convergent validity of the Thai DDS.

\section{Procedure}

Prior to data collection, the research protocol was submitted to and approved by the Institutional Review Board 
of Suranaree University of Technology according to the Declaration of Helsinki. Data were collected in October and November of 2013. Participants who met the eligibility criteria were then enrolled in the study. Both self-reported and face-to-face interviews were conducted by either the researchers or trained interviewers. Educated and knowledgeable participants were asked to complete the questionnaire on their own. A researcher assisted participants who were illiterate or had limitations by slowly reading the questions aloud and then asking them to rate each item. Participants were informed verbally about the purpose and protocols of the study, the time required for their participation, and that they could decline if they so wished. Confidentiality was maintained in all data collection and analyses, reports, and subsequent publications. While conducting test-retest reliability, the 30 diabetic seniors were asked to complete the questionnaire on two separate occasions within a 2 week period. The objectives of the method were explained to the participants. A second appointment for data collection was scheduled at the conclusion of the meeting.

\section{Data analysis}

Statistical analyses were performed using SPSS 14.0 software for Windows (SPSS Inc., Chicago, IL, USA). Descriptive statistics were used for demographic and clinical characteristics of the study sample. Five methods were used to test the psychometric properties of the Thai version of the DDS, namely: factor analysis, item analysis, internal consistency, convergent validity, and test-retest reliability.

An exploratory factor analysis (EFA) was conducted using SPSS 14.0 to determine the construct validity of the scale. Prior to EFA, the Kaiser-Meyer-Olkin (KMO) value was analyzed to determine whether the sample was adequate, and Bartlett's test was used to determine whether the correlation matrix was an identity matrix indicating that there was justification for factor analysis. ${ }^{24}$ EFA was conducted in two phases: a) a factor extraction method using principal component analysis (PCA) to derive independent subscales from the 17-item scale, and $b$ ) factor rotation using varimax methods in the extraction. Factor loading of $\geq 0.40$ was recommended to obtain practical significance of the items to be retained in each component. ${ }^{24}$

Item analysis was one of the statistical techniques used to investigate the pattern of responses to each item on the scale and provided information for revisions to improve the effectiveness of test items and validity of test scores. ${ }^{21}$ Following recommendations for item analysis as proposed by Ferketich ${ }^{25}$ and Nunnally and Bernstein, ${ }^{26}$ the 17 DDS items were examined. Three critical criteria were considered to help manage particular scale items. First, a cut-off value of 0.3 was used as an arbitrary guide for identifying valid items, which resulted in elimination of any item $<0.3$. Second, the inter-item correlation of all items was determined at $>0.2$; correlations between 0.3 and 0.7 are particularly recommended, which indicates no problems with redundancy. Third, the internal consistency estimate (alpha coefficient) was determined to ensure that it would not decrease if the item was deleted. ${ }^{25}$

For internal consistency analysis, this study used a Cronbach's alpha coefficient as a measure of scale reliability. Cronbach's alpha coefficient addresses the inter-relatedness of items on a scale; a high inter-item correlation suggests that the items could be measuring the same latent concept. ${ }^{27} \mathrm{~A}$ high correlation between items indicates that a scale is internally consistent. In the current study, both the subscales and the overall scale were assessed by Cronbach's alpha coefficient. A value of $\geq 0.70$ indicated sufficient internal consistency. ${ }^{26}$

To determine convergent validity, the Thai DDS-17 was compared with the Thai-GDS using Pearson's correlation coefficient. Our hypothesis was that diabetic patients with a high DDS would have high levels of depressive symptoms. ${ }^{4,5}$ The test-retest technique has been used for stability evaluation of a particular instrument. ${ }^{27}$ The 17 -item Thai-DDS was tested and re-tested within a 2 week interval. Data obtained from the two different tests were analyzed using the intraclass correlation coefficient (ICC) with confidence intervals (CI) of 95\%. The measurement is considered more stable the closer the CI is to $1.00 .^{19,24}$

\section{Results}

\section{Demographic and clinical data}

Demographic characteristics and clinical data of participants are listed in Table 1.

\section{Psychometric testing of the Thai-DDS Construct validity}

Thai-DDS data were examined for construct validity by PCA using a varimax rotation. Criteria used to determine the best fitting structure and correct numbers of factors were: 1) an eigenvalue $>1$; 2) screen plot characteristics; 3) percentage of total variance explained by each factor; 4) an item-factor loading cut-off of 0.40 ; and 5) a communality score cut-off of $0.50 .^{24,26,27}$ Results of the PCA with varimax rotation showed a KMO value of 0.93 , indicating sample adequacy for factor analysis. Bartlett's test of sphericity was 
Table I Participant sociodemographic characteristics and clinical data $(n=170)$

\begin{tabular}{|c|c|}
\hline Characteristics & Values \\
\hline \multicolumn{2}{|l|}{ Sex, n (\%) } \\
\hline Men & $54(31.8 \%)$ \\
\hline Women & $116(68.2 \%)$ \\
\hline Age, years, mean (SD) & $69(7.25 \%)$ \\
\hline \multicolumn{2}{|l|}{ Marital status, n (\%) } \\
\hline Single & $8(4.7 \%)$ \\
\hline Married & $101(59.4 \%)$ \\
\hline Widowed & $53(31.2 \%)$ \\
\hline Divorced & $8(4.7 \%)$ \\
\hline \multicolumn{2}{|l|}{ Education level, n (\%) } \\
\hline None & $33(19.4 \%)$ \\
\hline Primary school & II 4 (68.8\%) \\
\hline Secondary school and higher & $20(11.8 \%)$ \\
\hline \multicolumn{2}{|l|}{ Monthly income, n (\%) } \\
\hline No income & $12(7.1 \%)$ \\
\hline 500-I,000 Baht & $33(19.5 \%)$ \\
\hline $\mathrm{I}, 00 \mathrm{I}-2,000 \mathrm{Baht}$ & $39(23.1 \%)$ \\
\hline $2,00 \mathrm{I}-5,000 \mathrm{Baht}$ & $55(32.5 \%)$ \\
\hline$>5,000$ Baht & $31(17.8 \%)$ \\
\hline \multicolumn{2}{|l|}{ Living arrangement, n (\%) } \\
\hline Living alone & $18(10.6 \%)$ \\
\hline Living only with spouse & $28(16.5 \%)$ \\
\hline Living with children/relatives & $124(72.9 \%)$ \\
\hline Duration of diabetes, years, mean (SD) & $10.3(7.96)$ \\
\hline $\mathrm{HbAlc}$, mean (SD) & $7.8(4.95)$ \\
\hline \multicolumn{2}{|l|}{ Diabetes comorbidity, n (\%) } \\
\hline Hypertension & II 8 (69.4\%) \\
\hline Arthralgia/arthritis & $34(20.0 \%)$ \\
\hline Coronary heart disease & $15(8.8 \%)$ \\
\hline Kidney disease & 14 (8.2\%) \\
\hline Cerebrovascular disease & $8(4.7 \%)$ \\
\hline
\end{tabular}

Abbreviations: SD, standard deviation; HbAlc, glycated hemoglobin.

significant $\left(\chi^{2}=2536.42, P<0.001\right)$, indicating appropriateness of the data for further factor analysis. Results revealed three factors with eigenvalues $>1$ ranging from 10.14 to 1.27. Factor 1 included ten items $(1,3,5,6,8,10,11,12$, $14,16)$, namely emotional and regimen-related burden. Factor 2 included four items $(2,4,9,15)$, namely physicianand nurse-related distress, and Factor 3 included three items $(7,13,17)$, namely diabetes-related interpersonal distress. These components accounted for $59.63 \%, 7.67 \%$, and $7.46 \%$ of variance, respectively, and all components accounted for $74.76 \%$ of the total variance. The factor loading of the 17 -items ranged from 0.62 to 0.88 , with a statistical significance of $P<0.001$. The communality values ranged from 0.59 to 0.86 , indicating that all items were well reflected via their extracted factor. One item in the scale ("Feeling that I am not testing my blood sugars frequently enough") had a loading level $>0.50$ in more than one factor. This item was therefore moved to Factor 1, where it had the highest loading.
The reason for this move was that it was more directly related to the emotional burden of the diabetes regimen than physician- and nurse-related distress, as has been suggested by previous studies (Table 2). ${ }^{4,14,15}$

\section{Internal consistency}

Cronbach's alpha coefficients for all items of the ThaiDDS were 0.95 , indicating the instrument was reliable for measuring diabetes-related emotional distress. The mean inter-item correlation for the Thai-DDS was 0.56 ( minimum $=0.29$, maximum $=0.79$ ) and item-to-total correlations ranged from 0.55 to 0.85 . Cronbach alpha coefficients for the three subscales ranged from 0.85 to 0.96 , indicating high internal consistency. Mean, standard deviation, itemtotal correlation, and "alpha if item deleted" for each item are presented in Table 3.

\section{Convergent validity}

Convergent validity of the Thai-DDS was examined using Pearson's correlation coefficient between the Thai DDS-17 and the Thai-GDS. Findings showed that the ThaiDDS had a significant positive association with the Thai-GDS $(r=0.59, P<0.001)$ and the Thai-DDS subscales had significantly strong associations with the Thai-GDS ( $r=0.43-0.59$, $P<0.001$ ) (Table 4). As a result, the convergent validity of the Thai-DDS was confirmed.

\section{Test-retest reliability}

The intraclass correlation coefficient (ICC) with $95 \%$ confidence interval (CI) between scores of the two periods was 0.97 (95\% CI; 0.93-0.99), indicating excellent stability. In addition, correlations of the three subscales between Time 1 and Time 2 were as follows: 0.98 (95\% CI; 0.96-0.99) for diabetes-related interpersonal distress; 0.96 (95\% CI; 0.91-0.98) for emotional and regimen-related distress; and 0.89 (95\% CI; 0.75-0.95) for physician- and nurse-related distress. These results indicated that the 17-item Thai-DDS was stable over time.

\section{Discussion}

To our knowledge, this is the first study to translate and culturally adapt the original DDS-17 to a Thai version of the DDS in diabetic seniors and to evaluate its psychometric properties. Findings of the study demonstrate that the ThaiDDS is linguistically and culturally relevant to elderly Thai adults suffering from diabetes. In Stage 1 of the development of the Thai-DDS, a rigorous multi-stepped translation and back-translation process was undertaken that evolved into a 
Table 2 Factor loadings of the 17-item Thai-DDS for the three extracted factors after PCA with varimax rotation ( $n=170)$

\begin{tabular}{|c|c|c|c|c|}
\hline \multirow[t]{2}{*}{ Item } & \multicolumn{3}{|c|}{ Factor } & \multirow[t]{2}{*}{ Communality } \\
\hline & $\mathbf{I}$ & 2 & 3 & \\
\hline \multicolumn{5}{|l|}{ Factor I: Emotional and regimen-related burden } \\
\hline $\begin{array}{l}\text { I. Feeling that diabetes is taking up too much of my mental } \\
\text { and physical energy every day }\end{array}$ & 0.82 & 0.19 & 0.23 & 0.76 \\
\hline 8. Feeling that diabetes controls my life & 0.82 & 0.25 & 0.24 & 0.79 \\
\hline 10. Not feeling confident in my day-to-day ability to manage diabetes & 0.81 & 0.27 & 0.24 & 0.78 \\
\hline 14. Feeling overwhelmed by the demands of living with diabetes & 0.80 & 0.25 & 0.33 & 0.81 \\
\hline 6. Feeling that I am often failing with my diabetes routine & 0.80 & 0.34 & 0.14 & 0.77 \\
\hline 3. Feeling angry, scared, and/or depressed when I think about living with diabetes & 0.77 & 0.23 & 0.32 & 0.75 \\
\hline II. Feeling that I will end up with serious long-term complications, no matter what I do & 0.74 & 0.31 & 0.25 & 0.70 \\
\hline 12. Feeling that I am not sticking closely enough to a good meal plan & 0.70 & 0.36 & 0.30 & 0.71 \\
\hline 16. Not feeling motivated to keep up my diabetes self management & 0.65 & 0.32 & 0.42 & 0.70 \\
\hline 5. Feeling that I am not testing my blood sugars frequently enough & 0.62 & 0.51 & 0.14 & 0.66 \\
\hline \multicolumn{5}{|l|}{ Factor 2: Physician- and nurse-related distress } \\
\hline 9. Feeling that my doctor or nurse doesn't take my concerns seriously enough & 0.16 & 0.84 & 0.20 & 0.77 \\
\hline $\begin{array}{l}\text { 4. Feeling that my doctor or nurse doesn't give me clear enough directions } \\
\text { on how to manage my diabetes }\end{array}$ & 0.35 & 0.78 & 0.12 & 0.75 \\
\hline $\begin{array}{l}\text { 15. Feeling that I don't have a doctor or nurse who I can see regularly } \\
\text { enough about my diabetes }\end{array}$ & 0.31 & 0.70 & 0.31 & 0.68 \\
\hline $\begin{array}{l}\text { 2. Feeling that my doctor or nurse doesn't know enough about diabetes } \\
\text { and diabetes care }\end{array}$ & 0.35 & 0.67 & 0.15 & 0.59 \\
\hline \multicolumn{5}{|l|}{ Factor 3: Diabetes-related interpersonal distress } \\
\hline 17. Feeling that friends or family don't give me the emotional support that I would like & 0.27 & 0.14 & 0.87 & 0.86 \\
\hline 13. Feeling that friends or family don't appreciate how difficult living with diabetes can be & 0.29 & 0.20 & 0.85 & 0.85 \\
\hline $\begin{array}{l}\text { 7. Feeling that friends or family are not supportive enough of self-care efforts } \\
\text { (eg, planning activities that conflict with my schedule, encouraging me } \\
\text { to eat the "wrong" foods) }\end{array}$ & 0.34 & 0.33 & 0.76 & 0.79 \\
\hline
\end{tabular}

Note: Values in bold represent the highest factor loadings.

Abbreviations: DDS, Diabetes Distress Scale; PCA, principal component analysis.

version that preserved the meaning and content of the original instrument. ${ }^{28,29}$ Our theory-based translation procedure (back-translation model) ensured equivalence and prevented bias while using the Thai-DDS in elderly Thai adults with diabetes. As we translated the instrument, no items required significant modification to fit the Thai cultural context, except for adding a nurse along with a doctor in items 2, 4, 9, and 15 in the physician- and nurse-related distress component. In Thailand, diabetic patients cannot choose their own doctor, which could prove problematic with respect to the questions dealing only with physician-related relationships. Siripitayakunkit et $\mathrm{al}^{30}$ identified that Thai nurses play a central role in improving quality of care for diabetic patients, particularly by promoting self-management and integrating it into a patient's lifestyle. Adapting an existing instrument for cross-cultural study not only requires appropriate language translation; of equal importance, cultural and environmental contexts need to be considered as well. ${ }^{28,29}$

For psychometric testing, the 17-item Thai-DDS demonstrated consistent factor structure, high internal consistency, good validity, and high test-retest reliability. The factor structure of the Thai-DDS differed from that of the original DDS in that the emotional burden and regimen-related distress were included into the same factor. This discrepancy might be attributed to cultural differences of Thai seniors who perceive that living with diabetes requires lifelong treatment and self-management regimens, which might be closely related to emotional burden. Since most Thai seniors are poorly educated, have low literacy levels, and suffer from multiple chronic diseases, most depend on their families for social support and health care accessibility, both of which are significantly related to emotional well-being. ${ }^{31}$ However, Polonsky et $\mathrm{al}^{4}$ showed that the emotional burden (EB) and regimen-related distress (RD) aspects of the original DDS were highly correlated ( $r=0.88)$, suggesting that the two subscales were not totally independent. It is not surprising that in some factor analysis studies, some RD factor items were grouped with the EB factor. ${ }^{14,16}$ It can be concluded that the Thai-DDS factor model in the current study had a structure similar to the original DDS-17 and was able to meaningfully distinguish and measure three corresponding components of diabetes-related distress. 
Table 3 Item statistics for Factors I-3 of the Thai-DDS $(n=170)$

\begin{tabular}{|c|c|c|c|c|}
\hline Item & Mean & SD & $\begin{array}{l}\text { Item-total } \\
\text { correlation }\end{array}$ & $\begin{array}{l}\alpha \text { when item } \\
\text { deleted }\end{array}$ \\
\hline \multicolumn{5}{|l|}{ Factor I: Emotional and regimen-related burden $(\alpha=96)$} \\
\hline $\begin{array}{l}\text { I. Feeling that diabetes is taking up too much of my mental } \\
\text { and physical energy every day }\end{array}$ & 2.31 & 1.31 & 0.79 & 0.95 \\
\hline 8. Feeling that diabetes controls my life & 2.23 & 1.18 & 0.83 & 0.95 \\
\hline 10. Not feeling confident in my day-to-day ability to manage diabetes & 1.79 & 1.12 & 0.83 & 0.95 \\
\hline I4. Feeling overwhelmed by the demands of living with diabetes & 1.85 & 1.18 & 0.85 & 0.95 \\
\hline 6. Feeling that I am often failing with my diabetes routine & 1.79 & 1.03 & 0.80 & 0.95 \\
\hline $\begin{array}{l}\text { 3. Feeling angry, scared, and/or depressed when I think about } \\
\text { living with diabetes }\end{array}$ & 2.09 & 1.14 & 0.82 & 0.95 \\
\hline $\begin{array}{l}\text { II. Feeling that I will end up with serious long-term complications, } \\
\text { no matter what I do }\end{array}$ & 2.12 & 1.20 & 0.79 & 0.95 \\
\hline 12. Feeling that I am not sticking closely enough to a good meal plan & 1.70 & 0.94 & 0.81 & 0.95 \\
\hline 16. Not feeling motivated to keep up my diabetes self management & 1.54 & 0.84 & 0.80 & 0.95 \\
\hline 5. Feeling that I am not testing my blood sugars frequently enough & 1.42 & 0.68 & 0.74 & 0.95 \\
\hline \multicolumn{5}{|l|}{ Factor 2: Physician- and nurse-related distress $(\alpha=85)$} \\
\hline $\begin{array}{l}\text { 9. Feeling that my doctor or nurse doesn't take my concerns } \\
\text { seriously enough }\end{array}$ & 1.21 & 0.45 & 0.55 & 0.95 \\
\hline $\begin{array}{l}\text { 4. Feeling that my doctor or nurse doesn't give me clear enough } \\
\text { directions on how to manage my diabetes }\end{array}$ & 1.24 & 0.48 & 0.64 & 0.95 \\
\hline $\begin{array}{l}\text { 15. Feeling that I don't have a doctor or nurse who I can see } \\
\text { regularly enough about my diabetes }\end{array}$ & 1.21 & 0.51 & 0.65 & 0.95 \\
\hline $\begin{array}{l}\text { 2. Feeling that my doctor or nurse doesn't know enough } \\
\text { about diabetes and diabetes care }\end{array}$ & 1.23 & 0.51 & 0.60 & 0.95 \\
\hline \multicolumn{5}{|l|}{ Factor 3: Diabetes-related interpersonal distress $(\alpha=90)$} \\
\hline $\begin{array}{l}\text { 17. Feeling that friends or family don't give me the emotional } \\
\text { support that I would like }\end{array}$ & 1.42 & 0.83 & 0.61 & 0.95 \\
\hline $\begin{array}{l}\text { 13. Feeling that friends or family don't appreciate how difficult } \\
\text { living with diabetes can be }\end{array}$ & 1.50 & 0.90 & 0.65 & 0.95 \\
\hline $\begin{array}{l}\text { 7. Feeling that friends or family are not supportive enough of self-care } \\
\text { efforts (eg, planning activities that conflict with my schedule, } \\
\text { encouraging me to eat the "wrong" foods) }\end{array}$ & 1.51 & 0.84 & 0.71 & 0.95 \\
\hline
\end{tabular}

Note: $\alpha=$ internal consistency reliability.

Abbreviations: DDS, Diabetes Distress Scale; SD, standard deviation.

Internal consistency reliability was relatively high $(\alpha=0.95$ ), suggesting that reliability of the Thai version of the DDS was acceptable. This is consistent with recent results from the cross-cultural studies that found a relatively similar value of internal consistency for the DDS-17. ${ }^{14-16}$

The Thai-DDS demonstrated evidence of convergent validity, supporting the hypothesis that a higher DRD score is associated with greater scores in depressive symptoms. We found that the Thai-DDS was statistically and positively correlated to the Thai-GDS at a moderate level $(r=0.59, P<0.001)$. This supports previous studies that showed that DRD is moderately associated with depressive symptoms. ${ }^{1,414,15}$ To prevent depression in diabetes patients, the importance of assessing DRD as part of the medical management of diabetes is therefore recommended, as recommended in previous studies. ${ }^{1,4,15}$ However, some recent findings have suggested that high levels of DRD may not be significantly associated with major clinical depression. ${ }^{9,22}$ DRD was found to be more strongly and independently related to behavioral and clinical measures of diabetes management than was depression. ${ }^{9}$ Therefore, the need exists for initial screening for diabetes distress in diabetic patients in clinical settings.

Test-retest ICC was high (0.97), indicating that over time, the Thai-DDS had good consistency of test results. This result

Table 4 Pearson's correlation coefficients between the ThaiDDS and the Thai-GDS $(\mathrm{n}=170)$

\begin{tabular}{ll}
\hline Scale & Thai-GDS \\
\hline I. Emotional and regimen-related distress & $0.54^{*}$ \\
2. Physician- and nurse-related distress & $0.43^{*}$ \\
3. Diabetes-related interpersonal distress & $0.59^{*}$ \\
Entire Thai-DDS & $0.59^{*}$ \\
\hline
\end{tabular}

Note: $* p<0.001$.

Abbreviations: DDS, Diabetes Distress Scale; GDS, Geriatric Depression Scale. 
was greater than that found in the Norwegian DDS version $(r=0.76) .{ }^{15}$ This discrepancy may be due to the fact that the study used a 2 week period between the two measures of test-retest; respondents might have remembered their first test responses, thereby leading to over-estimation of reliability. ${ }^{21}$

There are some limitations to the current study that should be noted. First, since the sample was not randomly selected and was collected only in diabetes outpatient clinics of four community hospitals, generalization of the findings is limited. Second, study results might have been limited by a response bias by participants responding in a socially desirable manner (ie, answering the DDS questions on interpersonal distress was easier for elderly participants who were interviewed while surrounded by family members). Many studies on social desirability and culture have shown that populations from collectivistic societies such as Taiwan tend to respond in more socially desirable ways compared to more individualistic societies. ${ }^{32}$ Future use of the Thai-DDS should consider conducting a concurrent social desirability test to confirm that the scores on the scale are not biased in a socially desirable manner or influenced by significant others. Third, this study did not test the discriminant validity of the Thai-DDS. Thus, it may be premature to claim that this instrument can differentiate between diabetes patients in distress from those who are not. Fourth, construct validity using confirmatory factor analysis (CFA) has not yet been conducted. Further psychometric testing of the Thai-DDS using CFA would be of value in the future. Finally, the small sample size used to evaluate test-retest reliability may have affected the results of the stability evaluation of this instrument; users of the instrument should exercise caution with its use.

\section{Conclusion}

The Thai version of the DDS provides satisfactory results with respect to the process of translation and cultural adaptation and has acceptable psychometric properties. It allows for the assessment of diabetes-specific distress in elderly patients and has an advantage of being easy to use in both clinical and research settings.

\section{Acknowledgments}

This study was supported by a grant from the Foundation of Thai Gerontology Research and Development Institute, the Health Systems Research Institute (Thailand), and the National Research Council of Thailand.

\section{Author contributions}

KT was primarily responsible for study design, implementation, data collection, data analysis, data interpretation, and drafting the manuscript. WT and RK provided detailed guidance on study design and implementation, aided substantially in data interpretation, and contributed to drafting and editing the manuscript. All authors contributed equally to revising the manuscript and have approved the final version.

\section{Disclosure}

The authors report no conflicts of interest in this work.

\section{References}

1. Franks MM, Lucas T, Stephens MAP, Rook KS, Gonzalez R. Diabetes distress and depressive symptoms: A dyadic investigation of older patients and their spouses. Fam Relat. 2010;59:599-610.

2. Polonsky WH, Anderson B J, Lohrer PA, et al. Assessment of diabetesrelated distress. Diabetes Care.1995;18(6):754-760.

3. Fisher L, Skaff MM, Mullan JT, et al. Clinical depression versus distress among patients with type 2 diabetes. Diabetes Care. 2007;30(3): 542-548.

4. Polonsky WH, Fisher L, Earles J, et al. Assessing psychosocial distress in diabetes: development of the Diabetes Distress Scale. Diabetes Care. 2005;28(3):626-631.

5. Egede LE, Zheng D. Independent factors associated with major depressive disorder in a national sample of individuals with diabetes. Diabetes Care. 2003;26(1):104-111.

6. Cherrington A, Ayala GX, Sleath B, Corbie-Smith G. Examining knowledge, attitudes, and beliefs about depression among Latino adults with type 2 diabetes. Diabetes Educ. 2006;32(4):603-613.

7. Snoek FJ, Pouwer F, Welch GW, Polonsky WH. Diabetes-related emotional distress in Dutch and U.S. diabetic patients: cross-cultural validity of the problem areas in diabetes scale. Diabetes Care. 2000;23(9): 1305-1309.

8. Peyrot M, Rubin RR, Polonsky WH. Diabetes distress and its association with clinical outcomes in patients with type 2 diabetes treated with pramlintide as an adjunct to insulin therapy. Diabetes Technol Ther. 2008;10(6):461-466.

9. Fisher L, Glasgow RE, Mullan JT, Skaff MM, Polonsky WH. Development of a brief diabetes distress screening instrument. Ann Fam Med. 2008; 6(3):246-252.

10. Gonzalez JS, Fisher L, Polonsky WH. Depression in diabetes: have we been missing something important? Diabetes Care. 2011;34(1): 236-239.

11. Dunn SM, Smartt H, Beeney L, Turtle J. Measurement of emotional adjustment in diabetic patients: validity and reliability of ATT39. Diabetes Care. 1986;9(5):480-489.

12. Herschbach P, Duran G, Waadt S, et al. Psychometric properties of the questionnaire on stress in patients with diabetes - revised (QSD-R). Health Psychol. 1997;16(2):171-174.

13. Halter JB. Diabetes mellitus. In: Hazzard WR, Blass JP, Ettinger WH, et al, editors, Principles of geriatric medicine and gerontology. New York: McGraw-Hill; 1999:991-1011.

14. Ting RZW, Nan H, Yu MWM, et al. Diabetes-related distress and physical and psychological health in Chinese Type 2 diabetic patients. Diabetes Care. 2011;34(5):1094-1096.

15. Graue M, Haugstvedt A, Wentzel-Larsen T, Iversen MM, Karlsen B, Rokne B. Diabetes-related emotional distress in adults: reliability and validity of Norwegian versions of the Problem Areas in Diabetes Scale (PAID) and the Diabetes Distress Scale (DDS). Int J Nurs Stud. 2012; 49(2):174-182.

16. Joensen LE, Tapager I, Willaing I. Diabetes distress in type 1 diabetes - a new measurement fit for purpose. Diabetic Med. 2013;30(9): $1132-1139$.

17. Islam MR, Karim MR, Habib SH, Yesmin K. Diabetes distress among type 2 diabetic patients. Int J Med Biomed Res. 2013;2(2):113-124. 
18. Tol A, Baghbanian A, Sharifirad G, et al. Assessment of diabetic distress and disease related factors in patients with type 2 diabetes in Isfahan: A way to tailor an effective intervention planning in Isfahan-Iran. J Diabetes Metab Disord. 2012;11(1):20-24.

19. Burns N, Grove SK. The practice of nursing research: Appraisal, synthesis, and generation of evidence. 6th ed. St Louis: Saunders; 2009.

20. Lynn MR. Determination and quantification of content validity. Nurs Res. 1986;35(6):382-385.

21. Polit DF, Beck CT. Nursing research: principle and methods. Philadelphia: Lippincott Williams and Wilkins; 2004.

22. Fisher L, Hessler DM, Polonsky WH, Mullan JT. When is diabetes distress clinically meaningful?: establishing cut points for the Diabetes Distress Scale. Diabetes Care. 2012;35(2):259-264.

23. Thai Geriatric Depressive Scale. Siriraj Hosp Gaz. 1994;46:1-9. Article in Taiwanese.

24. Hair JF, Black WC, Babib BJ, Anderson RE, Tatham RL. Multivariate data analysis. 6th ed. New Jersey: Pearson Education, Inc.; 2006.

25. Ferketich S. Focus on psychometrics: Aspects of item analysis. Res Nurs Health. 1991;14(2):165-168.
26. Nunnally JC, Bernstein IH. Psychometric theory. 3rd ed. New York: McGraw-Hill; 1994.

27. DeVellis RF. Scale development: Theory and applications. 2nd ed. Thousand Oaks, CA: Sage; 2003.

28. Cha ES, Kim KH, Erlen JA. Translation of scales in cross-cultural research: issues and techniques. J Adv Nurs. 2007;58(4):386-395.

29. Curcio R, Alexandre NMC, Torres HC, Lima MHM. Translation and adaptation of the "Diabetes Distress Scale - DDS" in Brazilian culture. Acta Pal Enferm. 2012;25(5):762-767.

30. Siripitayakunkit A, Hanucharurnkul S, Melkus GD, Vorapongsathorn T, Rattarasarn C, Arpanantikul M. Factors contributing to integrating lifestyle in Thai women with type 2 diabetes. Thai J Nurs Res. 2008;12(3): $166-178$.

31. Knodel J, Chayovan N. Intergenerational relationships and family care and support for Thai elderly. Ageing Int. 2009;33:15-27.

32. Lalwani AK, Shavitt S, Johnson T. What is the relation between cultural orientation and socially desirable responding? J Pers Soc Psychol. 2006; 90(1):165-178.
Clinical Interventions in Aging

\section{Publish your work in this journal}

Clinical Interventions in Aging is an international, peer-reviewed journal focusing on evidence-based reports on the value or lack thereof of treatments intended to prevent or delay the onset of maladaptive correlates of aging in human beings. This journal is indexed on PubMed Central, MedLine,

\section{Dovepress}

CAS, Scopus and the Elsevier Bibliographic databases. The manuscript management system is completely online and includes a very quick and fair peer-review system, which is all easy to use. Visit http://www.dovepress. $\mathrm{com} /$ testimonials.php to read real quotes from published authors. 